\title{
Speckles and their dynamics for structured target illumination: optical spatial filtering velocimetry
}

Jakobsen, Michael Linde; Yura, H.T.; Hanson, Steen Grüner

Published in:

Journal of Optics. A. Pure and Applied Optics (Print Edition)

Link to article, DOI:

$10.1088 / 1464-4258 / 11 / 5 / 054001$

Publication date:

2009

Link back to DTU Orbit

Citation $(A P A)$ :

Jakobsen, M. L., Yura, H. T., \& Hanson, S. G. (2009). Speckles and their dynamics for structured target illumination: optical spatial filtering velocimetry. Journal of Optics. A. Pure and Applied Optics (Print Edition), 11(5), 9. https://doi.org/10.1088/1464-4258/11/5/054001

\section{General rights}

Copyright and moral rights for the publications made accessible in the public portal are retained by the authors and/or other copyright owners and it is a condition of accessing publications that users recognise and abide by the legal requirements associated with these rights.

- Users may download and print one copy of any publication from the public portal for the purpose of private study or research.

- You may not further distribute the material or use it for any profit-making activity or commercial gain

- You may freely distribute the URL identifying the publication in the public portal 


\title{
Speckles and their dynamics for structured target illumination: Optical spatial filtering velocimetry
}

\author{
M L Jakobsen ${ }^{1}$, H T Yura ${ }^{2}$ and S G Hanson ${ }^{1}$ \\ ${ }^{1}$ DTU Fotonik, Dept. of Photonics Engineering, P.O, Box 49, DK-4000 Roskilde, Denmark. \\ ${ }^{2}$ Electronics and Photonics Laboratory, The Aerospace Corporation, P.O. Box 92957, Los \\ Angeles, California, 90009 \\ E-mail: michael.linde.jakobsen@risoe.dk
}

\begin{abstract}
This contribution analyzes the dynamics of laser speckle patterns, designed for sensing with a receiver, based on spatial-filtering. Speckle translation is probed after freespace propagation of light scattered from non-specular moving surfaces. By structuring the target illumination the speckle patterns can be modulated with a fine structure, enhancing performance of the spatial filter. The space-time normalized cross-covariance function of speckle patterns incident on the spatial filter is presented for a simple structure of the field distribution on the target. This function provides the corresponding statistical parameters, i.e. structural size, velocity and decorrelation length, for both the speckles and the fine structure. The analysis also reveals that the speckles and the fine structure do not necessarily translate as a rigid structure. The spatial power spectrum for the speckle patterns and the spatial filter defines the optimum geometry for the structured illumination. The temporal power spectrum of the photocurrent from the optical spatial filter illustrates the direct influence of the speckle statistics on the sensor performance. Experimentally, a significant increase in signal level is obtained when illuminating the target with a structured field distribution, rather than using a single Gaussian beam. Moreover, the option of improving the signal quality by manipulating the target illumination will be discussed theoretically and experimentally.
\end{abstract}

Keywords: Optical sensor, displacement sensor, spatial filtering velocimetry, speckle translation, speckle interferometry.

\section{Introduction}

Methods based on analysis of speckle translation have successfully proven their operation [1,2] for measuring e.g. in-plane translation and out-of-plane rotation [3] of bodies with non-specular surfaces. Autocorrelation and cross-correlation functions $[1,4]$ can be applied to signals from single or multiple photodetector arrangements, and provide velocity measurements of speckle translation by correlation peak detection. Arrays of photodetectors or slit apertures enable spatial-filtering velocimetry $[1,2,5,6]$, where phase information of a filter-specific spatial-frequency component contained in the intensity distribution of speckle patterns is extracted to provide the velocity information. Particularly, differential-detection arrangements with phase-quadrature detection, based on transmission-grating [7], lenticular arrays [8] or detector-comb arrays [9], can provide high-performance velocity measurements based on simple signal processing algorithms such as zero-crossing detection, and thus facilitate real-time processing.

The optical spatial filter tracks in time the phase of ideally a single and narrow frequency component, present in the intensity distribution at the observation plane. The output of such an optical spatial filter, being the photocurrent $i(s)$, can be described as a function of speckle translation $s$ in one dimension via the definite integral of the product between the transmission function $T(p)$ of the spatial filter ( $p$ is position in the observation plane) and the incident speckle pattern intensity $I(p-s)$ given as:

$$
i(s)=\int_{-\infty}^{\infty} I(p-s) T(p) d p
$$


The spatial power spectrum of the photocurrent is given by $P_{i}(\xi)=P_{I}(\xi) P_{T}(\xi)$, where $P_{I}(\xi)$ is the spatial power spectrum of $I(p)$, and $P_{T}(\xi)$ is the spatial power spectrum of the transmission function. For an optical spatial filter with differential-detection, and with a rectangular-weighted, rectangulararray transmission function the spatial power spectrum can be written as [9]

$$
P_{T}(\xi)=\left(2 \xi_{0} D \Lambda_{1} w\right)^{2}\left\{\sum_{n=-\infty}^{\infty} \delta\left(\xi-(2 n+1) \xi_{0}\right) \times \operatorname{sinc}^{2}(w \xi)\right\} \otimes \operatorname{sinc}^{2}\left(D \Lambda_{1} \xi\right)
$$

where $\Lambda_{1}$ is the spatial period of the rectangular arrays, $w=\Lambda_{1} / 2$ is the width of the rectangles in the array ( $50 \%$ duty cycle), $D$ is the number of periods within the truncated transmission function (i.e. the width is $\left.D \Lambda_{1}\right), \xi_{0}=1 / \Lambda_{1}$ is the fundamental spatial frequency of the filter, and $\otimes$ indicates the convolution in (2). In figure 1 the spatial power spectrum of (2) is plotted. Clearly, with the option of modelling the spatial power spectrum of the speckle patterns, the more of the total optical power the designer can make coincide with the fundamental frequency $\xi_{0}$ of the optical spatial filter, the more efficient a sensor combining these technologies will be. Additionally, the signal-quality of the photocurrent in terms of suppression of higher harmonics can benefit significantly from an adequate design of the speckle spectrum. In practice, when implementing more complicated spatial filter, i.e. providing two photocurrents in phase quadrature, the second harmonic is not always sufficiently suppressed for e.g. zero-cross detection algorithms.

From the point of view of the spatial filtering velocimetry technology this paper will discuss both theoretically and experimentally the benefits of using structured illumination of the target in order to enhance signal power and signal quality.

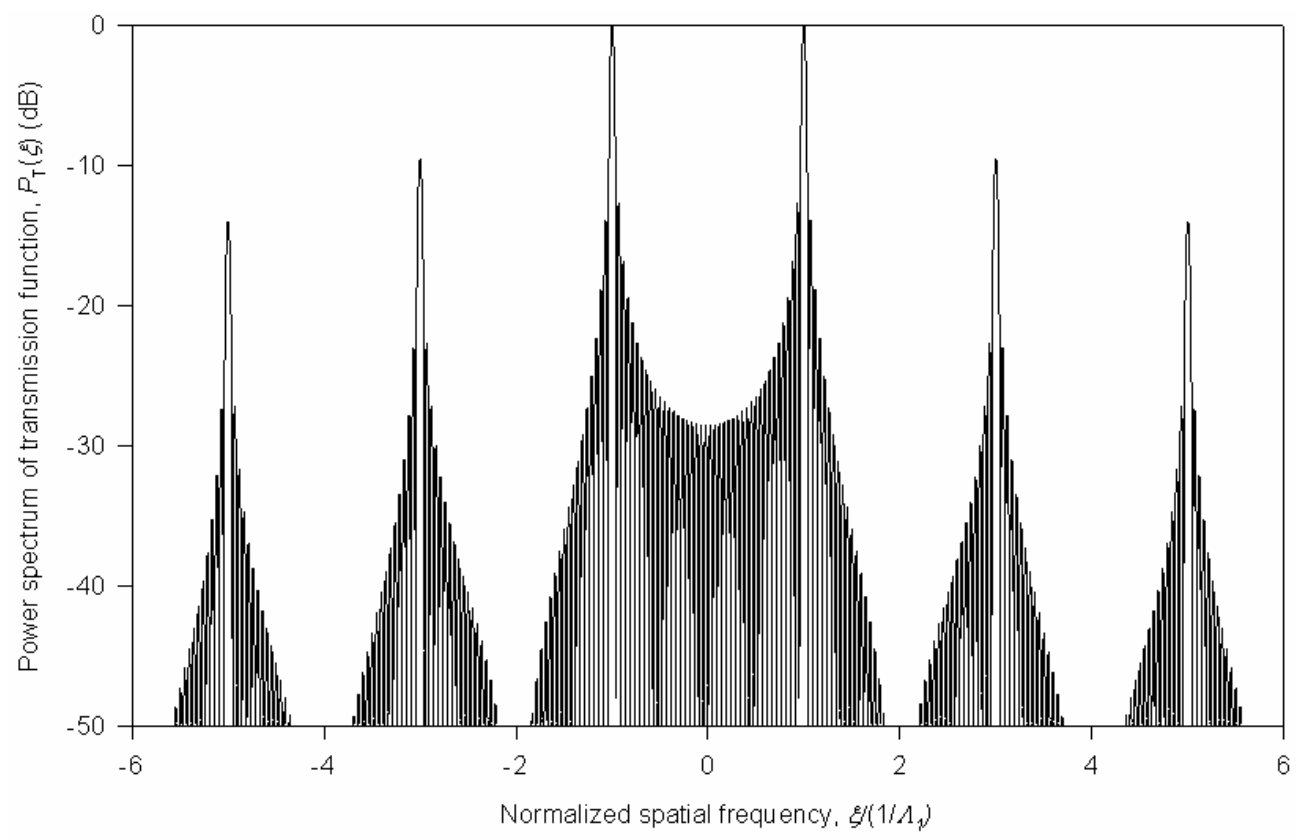

Figure 1. The spatial power spectrum is plotted for a rectangular-weighted rectangular-array transmission function with differential detection. For plotting (2) we use $D=20$.

\section{Theoretical review}

The speckle statistics and dynamics for light scattered from a target, which is illuminated by multiple, and mutually coherent beams, have been described analytically in terms of the space-time covariance of the light intensity, observed in the observation plane at a distance of $L$ from a moving target $[10,11]$. Within the Fresnel approximation of the Huygens-Fresnel diffraction we assume that the surface is sufficiently rough to form fully developed speckles at the observation plane. As illustrated in figure 2, we consider three Gaussian beams, originating from a shared point at a distance of $L^{\prime}$ from the target. The beams have relative angles of $\theta$ (aligned in one dimension), thus the target surface is illuminated with three separated spots, having equidistant spacing $\left(\Delta x=\theta L^{\prime}\right)$. In the observation plane a speckle pattern will appear, modulated with a spatially repetitive structure, which 
we will denote as the fine structure. The corresponding one-dimensional space-time covariance of the light intensities observed via free-space propagation at two positions in the observation plane $p$ separated by a distance of $\Delta p$ and a time lag of $\tau$, can be found in Reference [11]:

$$
\begin{gathered}
C_{I}(\Delta p ; \tau) \propto \exp \left(-\left(\frac{v \tau}{w_{0}}\right)^{2}-\frac{1}{\rho^{2}}\left[\Delta p-\left(1-\frac{L}{R}\right) v \tau\right]^{2}\right) \\
\left(1+2 \cos \left[k \theta \frac{L^{\prime}}{L}\left(\Delta p-\left(1+\frac{L}{L^{\prime}}\right) v \tau\right)\right]\right)^{2},
\end{gathered}
$$

where $v$ is the in-plane target velocity component along the direction of the three illuminating beams, $w_{0}$ is the beam radius at the target, $R$ is the radius of curvature of the beams (negative for a diverging beam) at the target. The distance of light propagation from the target surface to the observation plane is $L, k=2 \pi / \lambda$ is the optical wave number, and $\rho$ is the mean speckle size, given by $\rho=2 L /\left(k w_{0}\right)$. The exponential factor of the space-time covariance function describes the speckle statistics, while the cosine describes the fine structure. Accordingly, (3) provides a description of the speckle size $\rho$, the mean fringe spacing of the fine structure $(\lambda / \theta)$, the decorrelation length $\left(l_{\mathrm{d}}=w_{0}\right)$, and the dynamics for both structures. We define the gearing for each of the two structures as the ratio of structure translation to the corresponding mechanical translation of the target: $g_{i}=\Delta p / v \tau$. Therefore, the gearing for the speckle pattern is $g_{\mathrm{sp}}=(1-L / R)$, while the gearing for the fine structure is $g_{\mathrm{fs}}=\left(1+L / L^{\prime}\right)$. Thus, in general, the gearings for the speckles and the fine structure are different, except for the case where $L^{\prime}=$ $-R$.

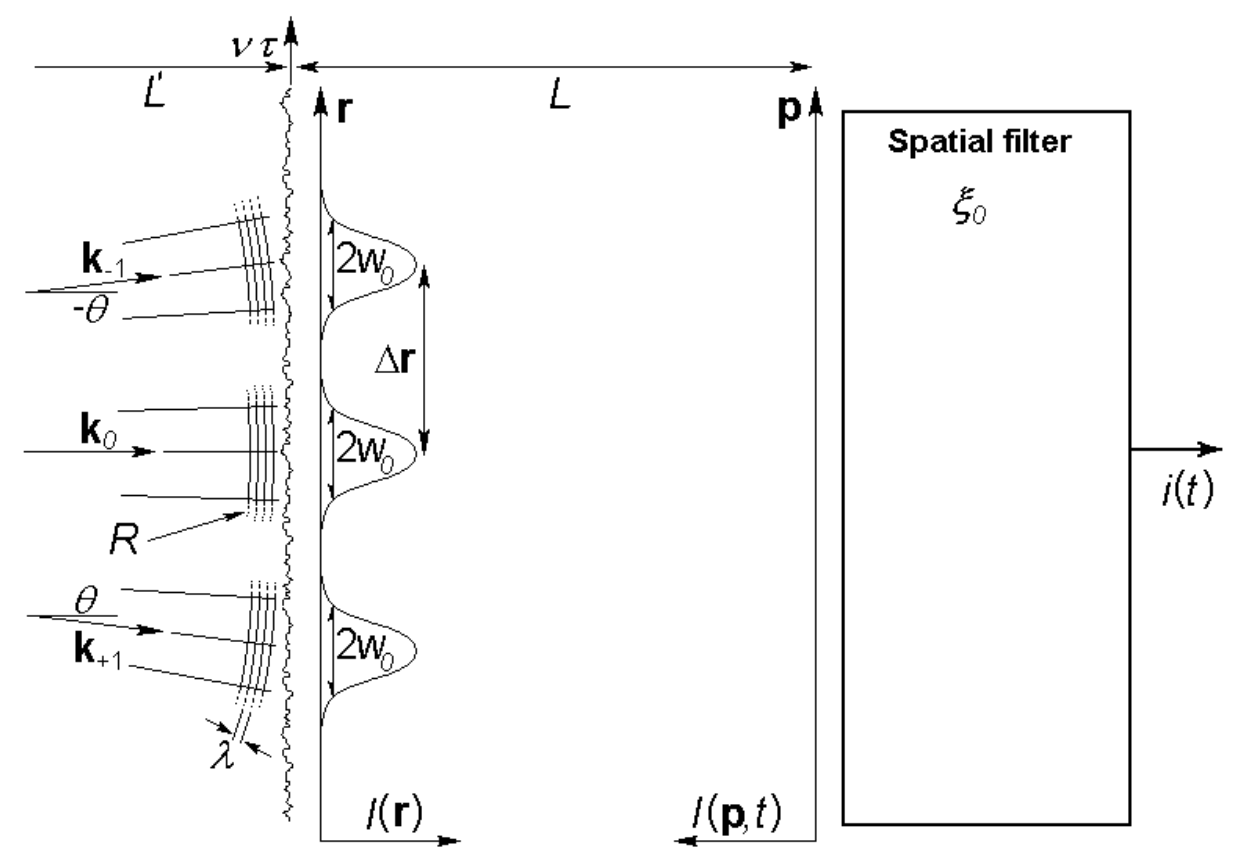

Figure 2. The schematic illustrates the three-beam illumination of the target, and the free-space propagation of speckles towards the observation plane at the spatial filter.

Considering the spatial correlation function of (1), inserting $\tau=0$ and Fourier transforming with respect to $\Delta p$, we find that the spatial power spectrum of the intensity distribution at the observation plane given by [10]:

$$
P_{I}(\xi)=6 \exp \left(-\frac{\xi^{2} L^{2}}{k^{2} w_{0}^{2}}\right)+4 \exp \left(-\frac{\left(\xi L \pm k \theta L^{\prime}\right)^{2}}{k^{2} w_{0}^{2}}\right)+2 \exp \left(-\frac{\left(\xi L \pm 2 k \theta L^{\prime}\right)^{2}}{k^{2} w_{0}^{2}}\right)
$$


where $\xi$ is the spatial frequency of the power spectrum. Figure 3 illustrates two power spectra presenting the cases of illuminating the target with one, and three beams. In case of single-beam illumination, the curves for two spot sizes are shown: $w_{0,1}=2 \theta \mathrm{L}, w_{0,2}=\theta \mathrm{L}$. The total optical power is the same for all three plots. Clearly, in case of three-beam illumination, power is "squeezed" from the surroundings into the fundamental frequency of the fine structure, at $\xi_{0}=k \theta L^{\prime} / L$. This occurs, however, without a similar increase in power in either the low frequency noise near $\xi=0$ or the higher harmonics of $\xi_{0}$. Therefore, the condition we are aiming for is a match between the fundamental frequencies of the fine structure and the spatial filter given by:

$$
\Lambda_{1}=\frac{\lambda L}{\theta L^{\prime}}
$$

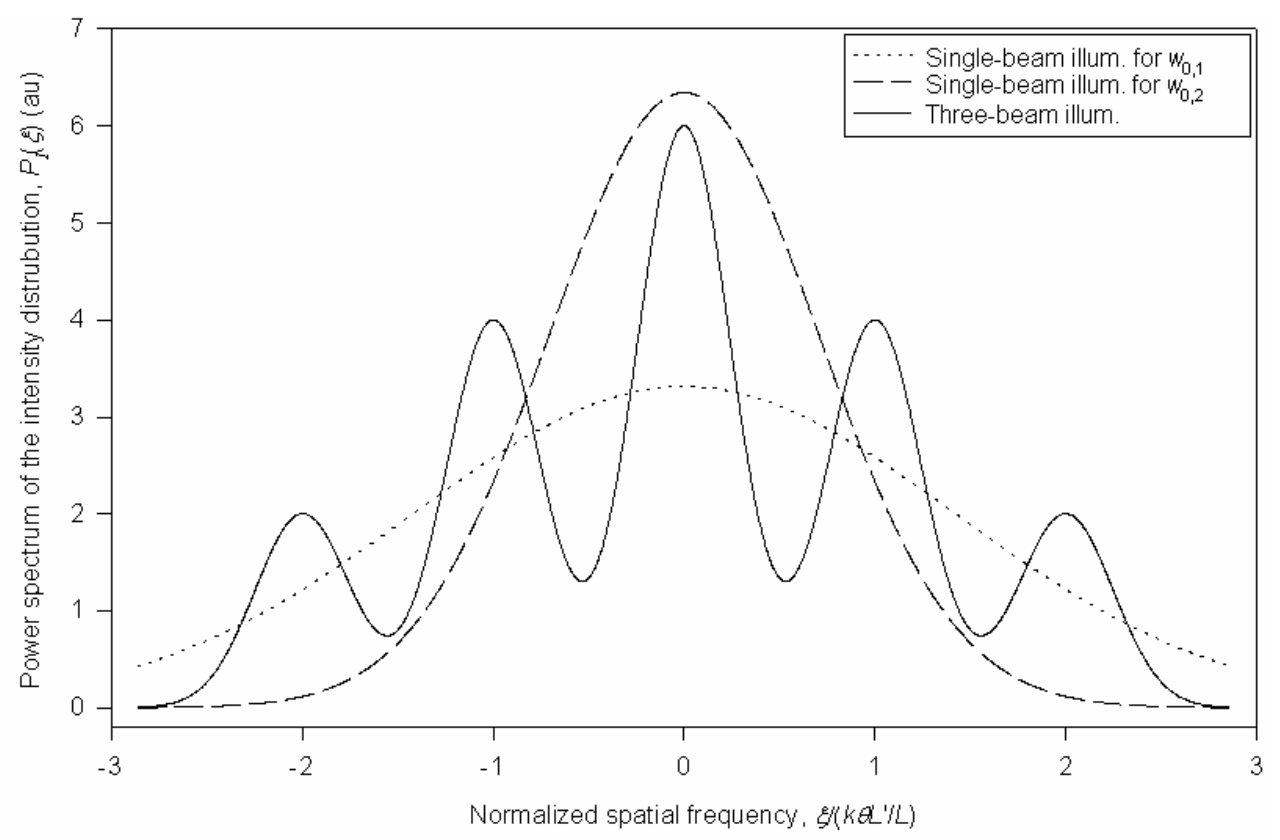

Figure 3. The ensemble averaged spatial power spectrum of an intensity distribution at the observation plane is illustrated for three-beam and single-beam illumination of the target. The singlebeam illumination is illustrated for two cases of spot sizes, $w_{0,1}=2 \theta \mathrm{L}$, and $w_{0,2}=\theta \mathrm{L}$.

The autocorrelation function and the power spectrum of the photocurrent from the optical spatial filter can be analysed in a similar way as the light intensity in the observation plane above. In Reference [10] the temporal photocurrent is used, and it is assumed that the rectangular array (as in (2)) of the transmission function for the spatial filter can be replaced with a cosine function. Physically, the transmission function is truncated by e.g. a Gaussian or a rectangular envelope. However, in practice the size of the lenticular array is much larger than the decorrelation length $\left(D \Lambda_{1}\right.$ $>>l_{\mathrm{d}}$ ), thus the influence of any envelope is negligible. The authors of Reference [10] find that the temporal power spectrum of the photocurrent, in case of for three-beam illumination, and assuming the case of (5), is given by: 


$$
\begin{aligned}
P_{i}(\omega) & =\exp \left[-\frac{(\omega-2 k \theta v)^{2}}{\omega_{0}^{2}}\right]+\frac{1}{2} \exp \left[-\left(\frac{3 L \theta}{w_{0}}\right)^{2}-\frac{\left(\omega-\left(4-3\left(1-\frac{L}{R}\right)\right) k \theta v\right)^{2}}{\omega_{0}^{2}}\right] \\
& +\exp \left[-\left(\frac{2 L \theta}{w_{0}}\right)^{2}-\frac{\left(\omega-2\left(1-\left(1-\frac{L}{R}\right)\right) k \theta v\right)^{2}}{\omega_{0}^{2}}\right] \\
& +\frac{3}{2} \exp \left[-\left(\frac{L \theta}{w_{0}}\right)^{2}-\frac{\left(\omega-\left(1-\frac{L}{R}\right) k \theta v\right)^{2}}{\omega_{0}^{2}}\right] \\
& +\frac{1}{2} \exp \left[-\left(\frac{L \theta}{w_{0}}\right)^{2}-\frac{\left.\left(\omega-\left(4-\left(1-\frac{L}{R}\right)\right) k \theta v\right)^{2}\right]}{\omega_{0}^{2}}\right] \\
+ & \operatorname{terms} \omega \rightarrow-\omega,
\end{aligned}
$$

where $\omega_{0}=2 v / w_{0}$. In figure 4 the temporal power spectrum of the photocurrent is plotted for $R=-L^{\prime}$, $R=-2 L^{\prime}$ and $R \rightarrow-\infty$. By substituting the rectangular array with a cosine function reveals that any higher harmonics in (6) vanish. This approximation allows for an elementary analytical solution, even though the experimental setups use rectangular arrays. The frequency of the fine structure is independent of $R$, and is illustrated in figure 4 for $R=-L^{\prime}$. The frequency of the speckles varies with $R$, and appears in the plots as the difference between the fundamental frequencies of the speckles and the fine structure, subsequently convolved with the fundamental frequency of the fine structure.

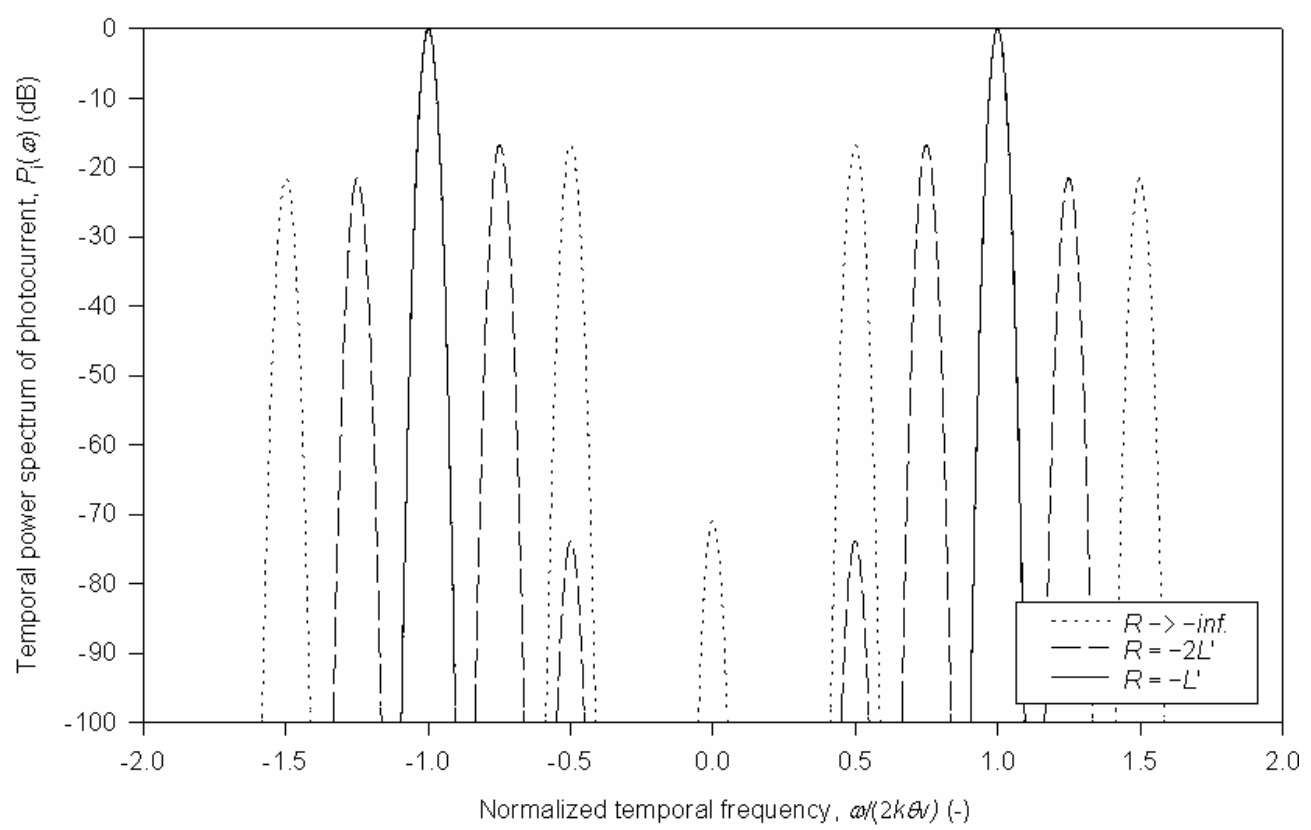

Figure 4. The temporal power spectra of the photocurrent plotted for $R=-L^{\prime}, R=-2 L^{\prime}$ and $R \rightarrow-\infty$.

As previously indicated, the gearings of the speckles and the fine structure are, in general, different. Thus, in case where the gearings are different, given the options for a sensor design, (6) 
illustrates the possibility to reduce all terms in (6), except for the first term, by selecting an adequately small value for $w_{0}$. Additionally, the peak width indicated in figure 3 will be proportional to $w_{0}$. Thus, by reducing $w_{0}$ correspondingly more of the available power will be moved to the vicinity of the fundamental peak of the spatial filter. Therefore, the power of the signal increases when minimising $w_{0}$. The expense for reducing $w_{0}$ is a similar reduction in decorrelation length $l_{\mathrm{d}}$, and therefore a broadening of the relative spectral width of the signal peaks in the temporal power spectrum. In other words, the speckle contribution can be eliminated by the spatial filter, while the fine structure enhances the signal related to the target motion.

\section{Optical setup}

The principle of this optical sensor technology, measuring in-plane surface motion in one dimension of a transparent target, is illustrated in figure 5. The transmitter illuminates the target with coherent light at a distance of $L^{\prime}$, and produces optionally a spatially modulated field distribution at the target. The transmitter includes a vertical cavity surface emitting laser (VCSEL), which emits light with a divergence angle of $\theta_{b}$, and is optionally preceded by different optical systems, which will be described in more detail below. The transparent target is a diffuser, which can move in the plane perpendicular to the optical axis of the sensor. The light scattered from the surface propagates in free space a distance $L$ to the entrance plane of the receiver. Accordingly, the mechanical target motion will be restricted to in-plane translation only.

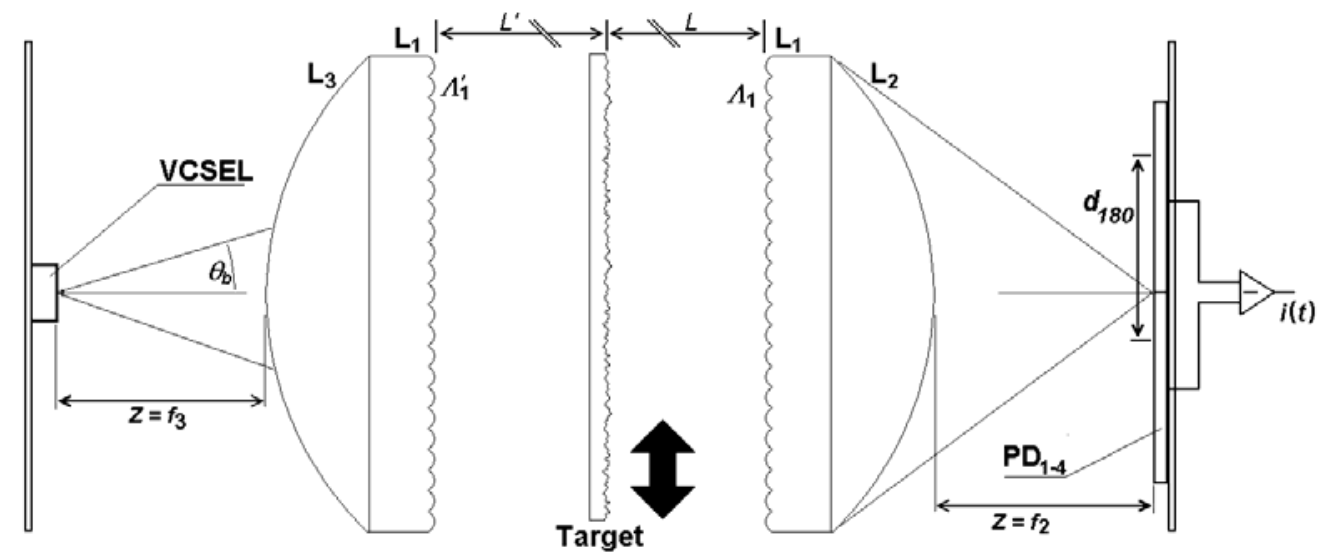

Figure 5. Illustration of the principle of the transmitter (left) and the receiver (right), arranged in-line as a forward-scattering system.

The spatial filter is implemented using a lenticular array $\left(\mathrm{L}_{1}\right)$ with focal length $f_{1}$, with pitch $\Lambda_{1}$, and a spherical lens $\left(\mathrm{L}_{2}\right)$ with focal length $f_{2}$. In the detector plane a differential pair of photodetectors is positioned with a centre spacing of $d_{180}$. For a successful implementation of an optical spatial filter both with differential-detection, and a rectangular-weighted rectangular-array transmission function (2), the optics must fulfil the following relation [12]:

$$
d_{180}=\frac{\Lambda_{1} f_{2}}{2 f_{1}} .
$$

Two transmitter designs are considered, where one modulates the intensity distribution at the target. In this case, the beam from the VCSEL is collimated with a lens $\mathrm{L}_{3}$, of a focal length of $f_{3}$. Then, the beam passes a diffractive optical element (DOE), with a regular pitch or period of $\Lambda_{1}^{\prime}$. In this case, in the far field of the diffracted beam, the intensity distribution appears as mainly three separate spots, having a fundamental spacing of $\Delta x=\lambda L^{\prime} / \Lambda_{1}{ }^{\prime}$, where $\theta=\lambda / \Lambda_{1}{ }^{\prime}$, a spot radius given by $w_{0}=f_{3} \tan \left(\theta_{6}\right)$ and a radius of curvature of $R \rightarrow-\infty$. Therefore, in this case the gearing for the speckles is close to a factor of unity $\left(g_{\mathrm{sp}} \approx 1\right)$, while the gearing for the fine structure is a factor of two $\left(g_{\mathrm{fs}}=2\right)$. In the standard setup we have not tried to eliminate the speckle contribution. Therefore, it is present but weaker than the contribution from the fine structure 
In the alternative transmitter design, the VCSEL illuminates the target directly, with the propagated beam profile. In this case of using a single mode VCSEL, the power spectrum of the speckle pattern at the receiver will be a Gaussian function, as it is illustrated in figure 3 for singlebeam illumination, with a beam diameter given as $2 L^{\prime} \tan \left(\theta_{\mathrm{b}}\right)$. The radius of curvature for the beams is given by $R \cong-L^{\prime}$ (the confocal parameter is $0.1 \mathrm{~mm}<<L^{\prime}$ ). In this case, the working distance $\left(L^{\prime}\right)$ is given by the distance the emission window of the VCSEL to the target.

\section{Experimental setup}

The experimental data are obtained, using the following relevant specifications for the optical filter: $\Lambda_{1}=15.0 \mu \mathrm{m}$. The VCSEL dye (Truelight: TSD-8A12) emits a transversal multimode beam at an optical wavelength of $\lambda=850 \mathrm{~nm}$, and a divergence angle of $\theta_{0} \approx 8^{\circ}$ (i.e. FWHM). The DOE design tested within this work is a crossed lenticular array (2-dimensional), where $\Lambda_{1}{ }^{\prime}=15.0 \mu \mathrm{m}, f_{1}{ }^{\prime}=30 \mu \mathrm{m}$ and $f_{3}=0.78 \mathrm{~mm}$. A typical diffractive intensity distribution generated by the DOE in the far field is illustrated in figure 6 . The alternative transmitter is a sensor, where the collimation lens $\left(\mathrm{L}_{3}\right)$ and the DOE are removed, leaving the VCSEL dye, illuminating the target directly.

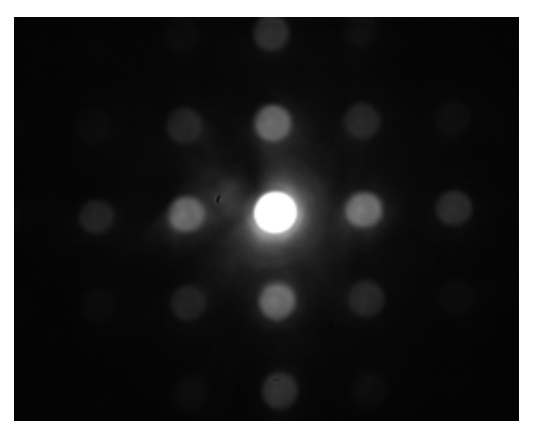

Figure 6. The intensity distribution acquired at the target is illustrated for the DOE used in this work.

The diffuser is a $1 \mathrm{~mm}$ thick grounded glass plate. The target is translated at a constant speed of e.g. $v$ $=0.74 \mathrm{~mm} / \mathrm{s}$ with a linear translation stage, and will accordingly produce quasi-sinusoidal sensor signals with a fundamental frequency of approximately $f=\left(1+L / L^{\prime}\right) v / \Lambda_{1} \approx 2 v / \Lambda_{1}=100 \mathrm{~Hz}$. The signals are acquired at a sampling rate of $2.0 \mathrm{kS} / \mathrm{s}$, and in time-records of $10^{5}$ samples. The mean frequency of the fundamental signal peak is determined from ensemble-averaged power spectra of time-records using the first and second moments. Typically, 100 power spectra contribute to an ensemble-averaged power spectrum. The power contained in a given fundamental signal peak is determined by integrating the ensemble-averaged power spectrum through an interval of a length corresponding to three times the estimated peak width and centred at the peak position. The signal level obtained from the receiver is normalized to the total optical power emitted by the transmitter, and the square of this ratio is denoted by normalized-signal-power.
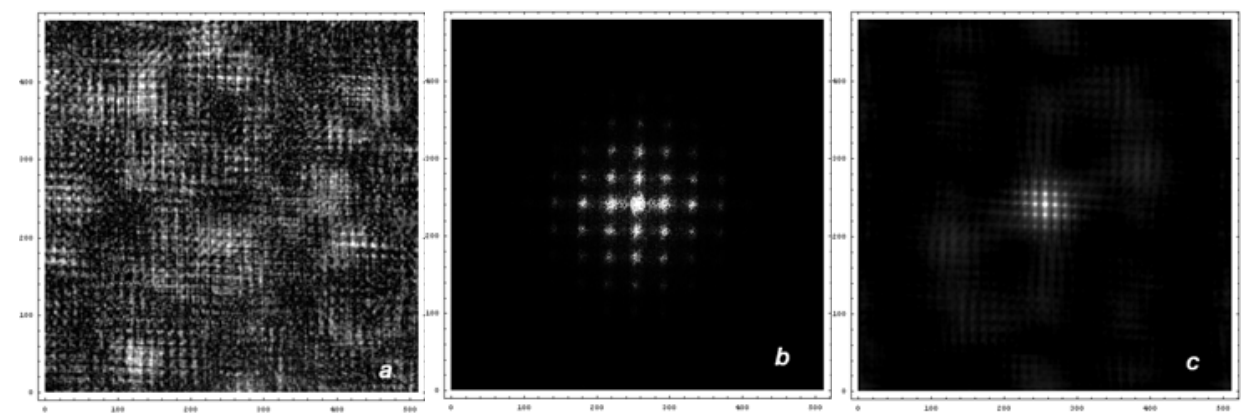

Figure 7. The images illustrate the intensity distribution (a), the power spectrum (b) and the autocorrelation function (c) of a speckle pattern observed at the observation plane. 


\section{Results}

The intensity distribution of a speckle pattern, formed by a modulated target illumination is captured with a CCD camera located at the observation plane. Figure 7 illustrates the intensity distribution, its spatial power spectrum and the corresponding autocorrelation function. Clearly, the fine structure modulates the speckle pattern, the power spectrum illustrates the five main peaks from figure 3 (in 1dimension), and the autocorrelation function illustrates that the phase of the fine structure is correlated only within lengths of the order of the mean speckle size.

\subsection{Matching the fine structure.}

The signal power obtained within the fundamental peak in the power spectrum is determined as a function of $L^{\prime}$ for various $L$-values. In figure 8 such normalized-signal-power characteristics are plotted for three different $L$-values. For the transmitter with a DOE, the characteristics obtained at $L=$ $4 \mathrm{~mm}$ is plotted, and clearly the importance of a good match between the fine structure and the pitch of the spatial filter of the receiver (5) is demonstrated with the peak at $L^{\prime}=4 \mathrm{~mm}\left(\Lambda_{1}=\Lambda_{1}^{\prime}\right)$. Furthermore, an additional peak appears at $L^{\prime}=2 \mathrm{~mm}$ where the second harmonic of the diffraction pattern at the target can seed the speckle patterns with the adequate spatial frequency $\xi_{0}$.

For the transmitter without a DOE the normalized-signal-power characteristics obtained at $L$ $=2 \mathrm{~mm}, 4 \mathrm{~mm}$ and $8 \mathrm{~mm}$ are plotted, and as expected, these curves do not demonstrate a strong selectivity for variations in $L^{\prime}$. However, the characteristics demonstrate a strong drop-off for $L^{\prime}<$ $0.7 \mathrm{~L}$. This drop-off occurs because the width of the speckle spectrum is proportional to the spot size $w_{0}$ at the target, and therefore, proportional to $L^{\prime}$. Thus, by reducing the width of the speckle spectrum below the fundamental frequency of the spatial filter $\xi_{0}$ the normalized-signal-power will decrease rapidly. The slowly drop in signal for $L^{\prime}>L$ is due to the limited field of view of the receiver as the illuminated area increases with $L^{\prime}$.

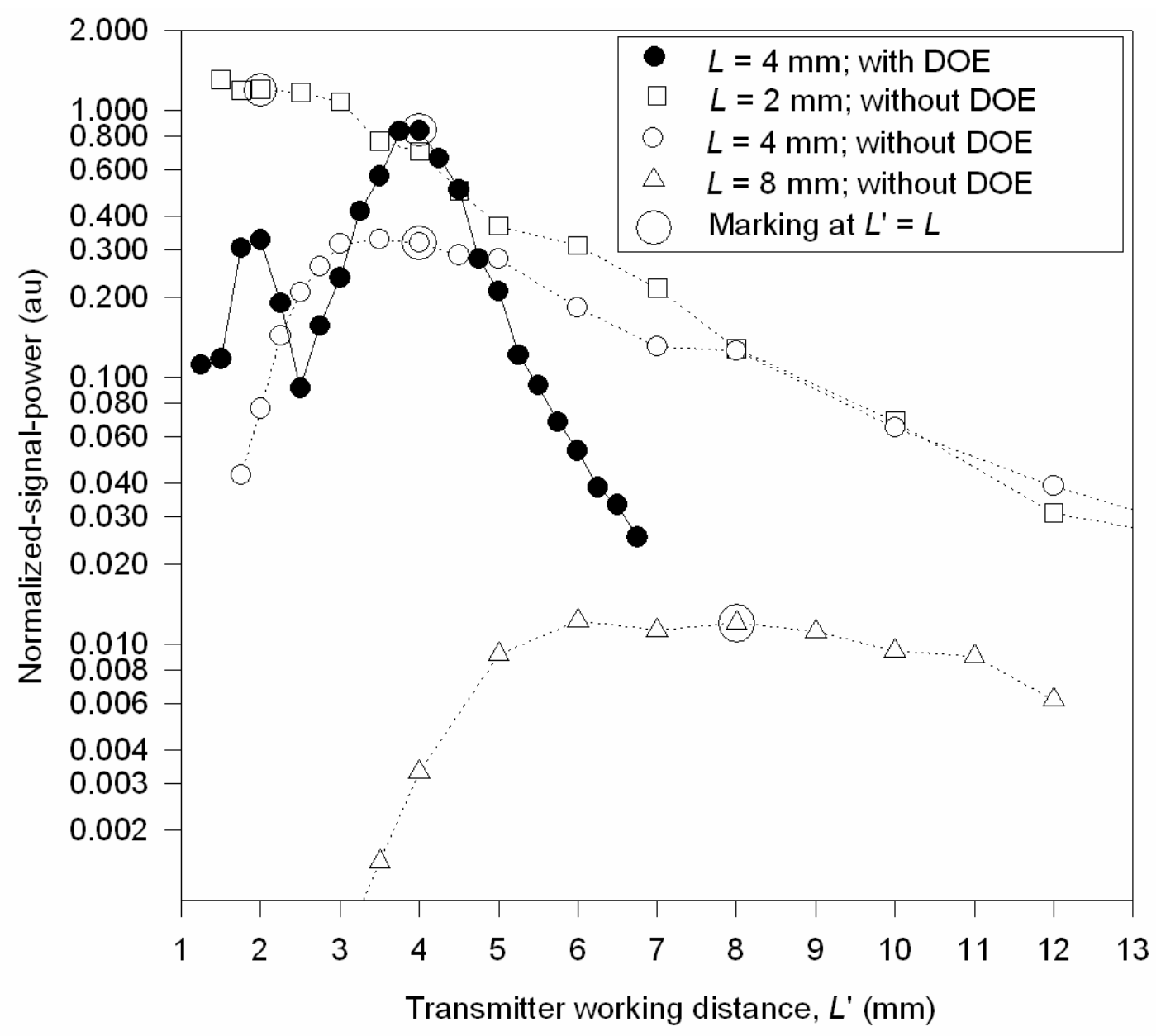

Figure 8. The normalized-signal-power is plotted as a function of $L^{\prime}$ for a transmitter with and without a DOE, and at various $L-$ values of the receiver. 


\subsection{Gain in signal power}

In the case of using a transmitter with a DOE, the observed peak for the normalized-signal-power in figure 8 is a reliable indication for the match between the fundamental frequencies of the fine structure and of the spatial filter $\left(L^{\prime}=L\right)$. In case of using a transmitter without DOE, the matching points are determined at a constant target velocity by tuning the signal frequency to the signal frequency measured for the transmitter with DOE for the condition of match $\left(L^{\prime}=L\right)$. Figure 9 shows the normalized-signal-power measured at these matching points plotted as a function of $L$ for cases of using a transmitter with and without a DOE. Clearly, the curves indicate that at $L>3 \mathrm{~mm}$, the sensor with the given transmitter DOE gains a factor of about 3-8 in normalized-signal-power, as compared to the given sensor operating without a transmitter DOE.

The curves depict a continuous decrement in normalized-signal-power using both transmitters as the working distance increases from 3 to $10 \mathrm{~mm}$. This relationship is primarily due to the decreasing light-collection angle of the receivers with increasing working distance. The signal power is expected to be proportional to $L^{-4}$, and this is confirmed when $L>4 \mathrm{~mm}$ for the transmitter without a DOE by observing that the normalized-signal-power is proportional to $L^{-3.9}$. For the transmitter with a DOE the trend is similar though we measure that the corresponding signal power is proportional to $L^{-2.9}$.

The curve for a transmitter without a DOE follows a constant trend throughout the entire range of working distances. However, for $L<3 \mathrm{~mm}$ the curve for a transmitter with DOE, experiences saturation in the normalized-signal-power. At $L \approx 1.5 \mathrm{~mm}$ the two curves intersect.

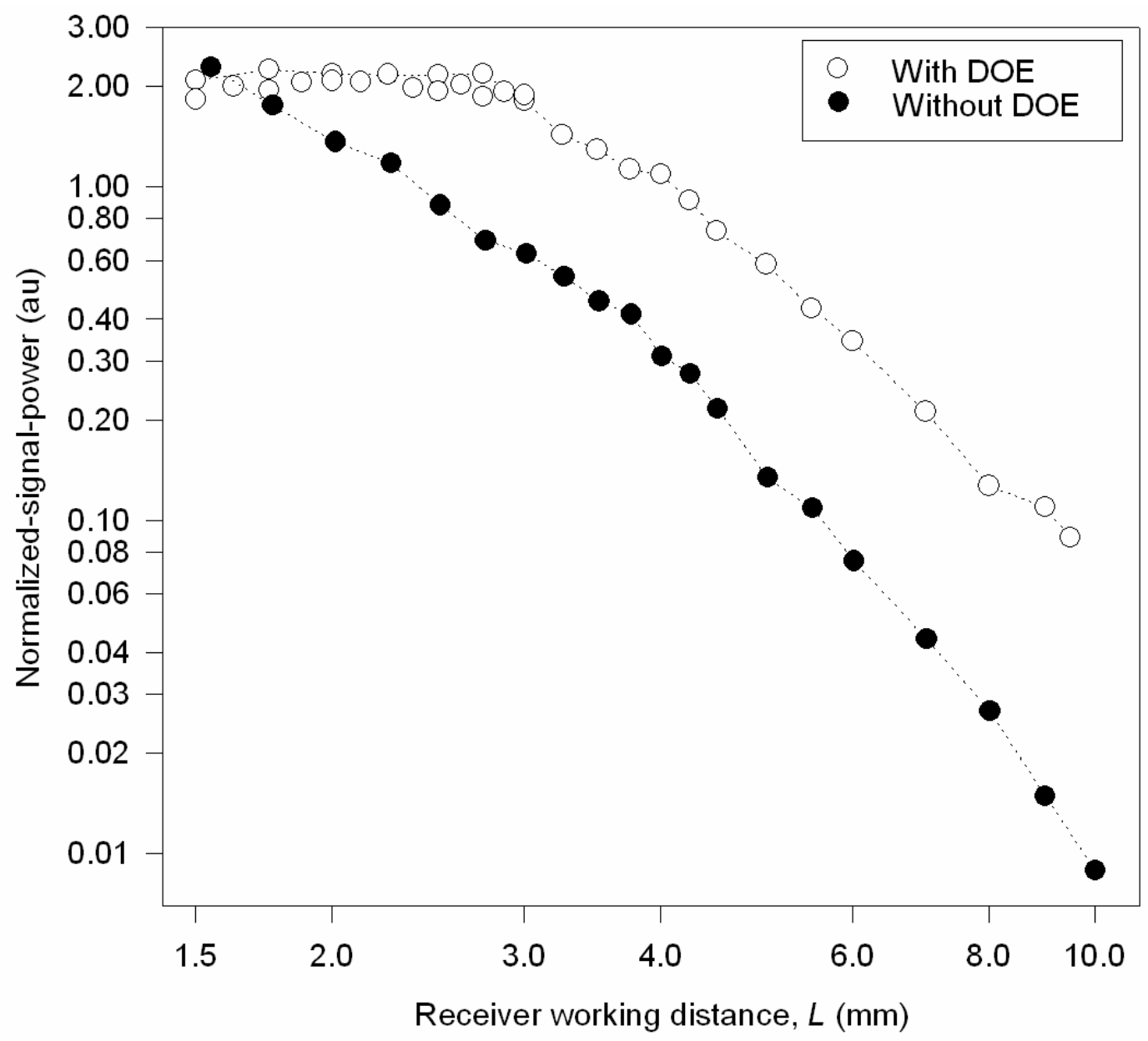

Figure 9. The figure illustrates the normalized-signal-power, obtained at the matching conditions, and plotted as a function of $L$. The data points are acquired using both a transmitter with and without a

DOE. 


\section{Discussion}

The difference in gearing between the speckles and the fine structure for the sensor having a transmitter with a DOE can be observed in figure 10. The setup used for this measurement is slightly different from the setup described above. The same transmitter and receiver are integrated in one optical component located side-by-side on the same side of the target. Therefore, this sensor is operated in a backscattering configuration, and is targeting a non-transparent moving object. The centre distance between the transmitter and the receiver is $1.0 \mathrm{~mm}$, and $L^{\prime}=L$.

In the power spectrum of the photocurrent we observe the highest peak at $675 \mathrm{~Hz}$, which corresponds to a gearing of a factor of $2.0 \pm 0.01$, and is caused by the dynamics of the fine structure. The peak level increases with $L$ just as observed in figure 10, and remain independent of variations in $R$ which can be introduced by detuning the collimation lens $f_{3}$ in front of the DOE. The fine structure also introduces a second harmonic, located at $1350 \mathrm{~Hz}$.

We observe a weaker peak at $338 \mathrm{~Hz}$, and its mirrored peak at $975 \mathrm{~Hz}$. The first peak corresponds to a gearing of a factor of unity and is caused by the speckle dynamics. When, increasing $L$ we observe a fast drop in the levels of these peaks. When, varying $L$ the spot size at the target $w_{0}$ is nearly constant because the beams are nearly collimated. Therefore, according to (6) all terms vanish for an increasing $L$ except the first term, describing the fine structure. Additionally, the position of these peaks depends on $R$.

The fine structure provides a signal significantly stronger than the speckles. Therefore, in the remainder of this paper we will refer to the fundamental peak caused by the fine structure as the sensor signal.

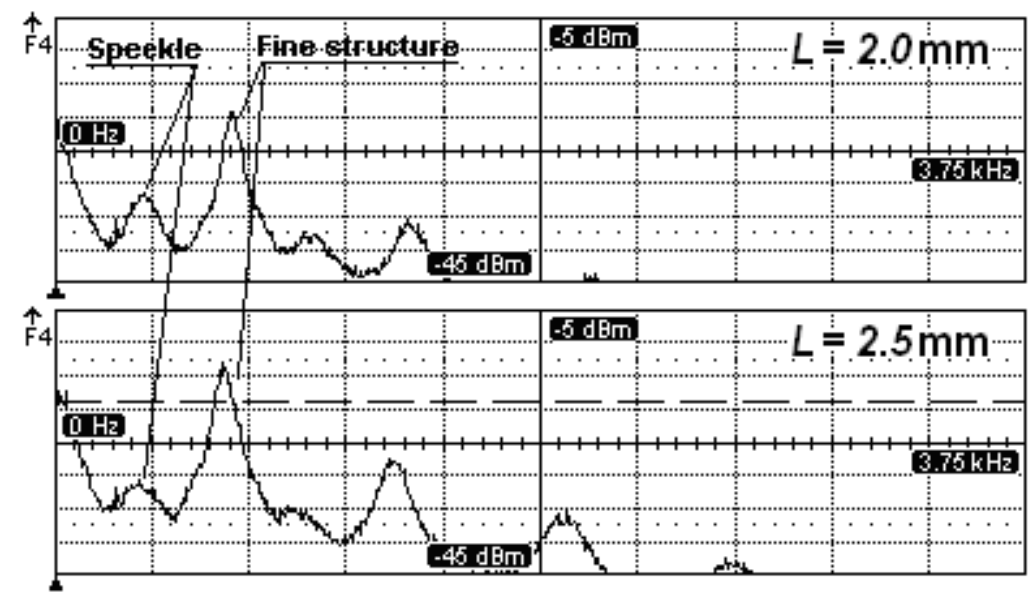

Figure 10. The speckles and the fine structure provide different signal frequencies. Further, the two plots illustrate that the power of the speckle contribution decreases with $L$, while the power of the fine structure increases with $L$.

As we discussed below (6) the speckle contribution can be eliminated by minimizing $w_{0}$. Therefore, by detuning the collimation lens $f_{3}$ we can make the beams converge towards the target. At $0.1 \mathrm{~mm}$ offset of the VCSEL away from the lens $f_{3}$ we find that the speckle contribution disappears at $L \geq 4 \mathrm{~mm}$. The additional enhancement of the signal and the broadening of the width of the peaks in the speckle spectrum $P_{\mathrm{i}}(\omega)$, by reducing the size of $w_{0}$, is observed as well.

Experimentally, we observe in the power spectrum in figure 10 that the second harmonic of the signal is attenuated by approximately $15 \mathrm{~dB}$ relative to the fundamental part of the signal. Apparently, in the case of three-beam illumination the second harmonic of the signal is still present. Therefore, the suppression of the second harmonic in the power spectrum of the spatial filter (i.e. (2)) can not be perfect. We believe that this imperfection is caused by a non-uniform mean intensity distribution over the entire detector area. In the case of the backscattering setup two sets of differential pairs of photodetectors, are mutually shifted distance $d_{180} / 2$ (four detectors with $w=d_{180} / 2$ ) to provide two photocurrents in phase quadrature. Therefore, it is not possible to balance both of the two differential detector pairs, and that means that the suppression of both low-frequency and second harmonic is reduced, as compared to figure 1. In particular, the second harmonic can distort the phase of the signal and cause problems with synchronized zero-cross detection of phase quadrature signals. 
In this case one might consider a dual-beam illumination to enhance the suppression of the second harmonic. Two beams incident on the target will produce a spatial power spectrum $\left(P_{\mathrm{I}}(\xi)\right.$, see figure 3) for the speckles with three peaks located at $\xi=0$ and $\xi_{0}= \pm \theta / \lambda I$ (see (5)). Therefore, the second harmonic will not be present in the fine structure, and additional suppression of the second harmonic in the photocurrent will be achieved.

In figure 11 we have plotted the power spectrum for the photocurrent obtained from a sensor where the DOE, providing the $3 \times 3$ beams on the target, is replaced with an element producing $2 \times 2$ beams on the target and with the same spacing $\Delta x$ between the spots. Clearly, the additional suppression of the second harmonic by the transmitter has eliminated the second harmonic almost entirely from the power spectrum, to a level relative to the fundamental of better than $-30 \mathrm{~dB}$.

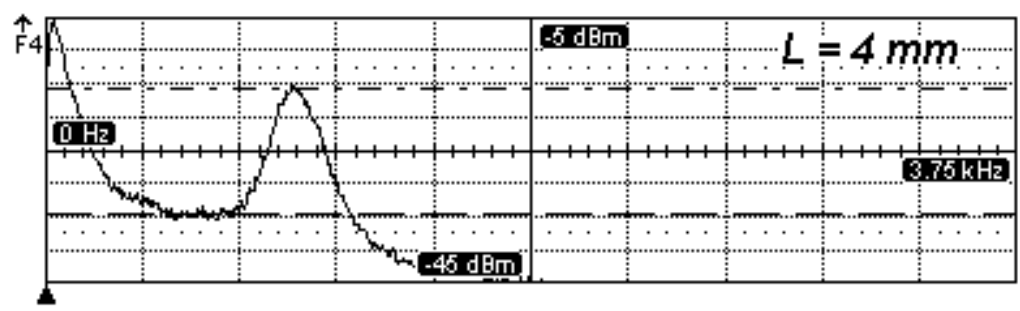

Figure 11. The figure illustrates the power spectra of the photocurrent for the case of dual-beam illumination of the target.

Clearly, the plots in figure 8 illustrate the selectivity of the spatial filtering of the transmitter and the receiver as they are detuned mutually. If we recalculate (6), but without the condition of match between the fundamental frequencies of the transmitter and the receiver (i.e. (5)), we obtain that the peak power of the fundamental frequency, $\omega_{0}=k \theta v\left(1+L / L^{\prime}\right)$ as a function of $L^{\prime}$ is given by:

$P_{i}\left(L^{\prime}\right)=\exp \left[-\left(L^{\prime}-L\right)^{2} \theta^{2}\left(\frac{4 L^{\prime 2}+k^{2} w_{0}^{4}}{4 L^{\prime 2} w_{0}^{2}}\right)\right]+\frac{1}{2} \exp \left[-\left(2 L^{\prime}-L\right)^{2} \theta^{2}\left(\frac{4 L^{\prime 2}+k^{2} w_{0}^{4}}{4 L^{\prime 2} w_{0}^{2}}\right)\right]$.

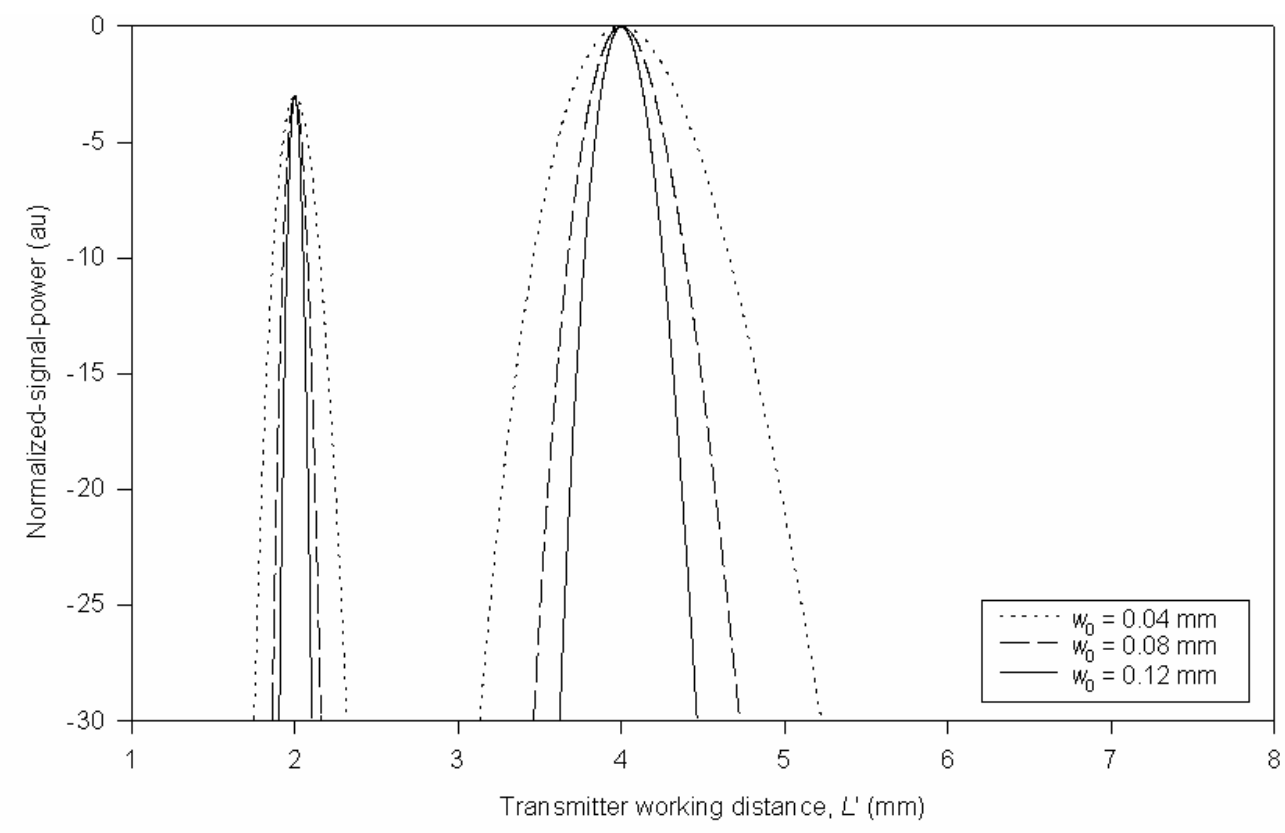

Figure 12. The theoretical peak power of the fundamental signal frequency is plotted as a function of working distance of the transmitter, $L^{\prime}$. Various beam radii have been inserted: $w_{0}=0.04 \mathrm{~mm}, w_{0}=$ $0.08 \mathrm{~mm}$ and $w_{0}=0.12 \mathrm{~mm}$.

In figure 12 the expected selectivity has been plotted as a function of $L^{\prime}$ for various beam radii $\mathrm{w}_{0}$, using similar parameters that are used in the experimental setup that led to figure 8 . The present 
theory does not include the drop in signal level, due to the limited field of view of the spatial filter. The peak level at $L^{\prime}=2 \mathrm{~mm}$ is $40 \%$ of the peak level at $L^{\prime}=4 \mathrm{~mm}$, and this is in good agreement with figure 8 . However, the widths of the peaks in figure 12 are significantly narrower than they are in figure 8 . The model can not explain this deviation, thus we expect that either the approximations that led to (6) or the imperfections of the experimental setup are be responsible.

Figure 9 illustrates the signal power as a function of working distance for $\left(L=L^{\prime}\right)$, and the corresponding enhancement of the signal when introducing the fine structure to the speckle pattern. However, it also illustrates that the effect of the fine structure decreases with working distance for $L<$ $3 \mathrm{~mm}$ for the given sensor design. Considering diffraction angles of $3^{\circ}$ and a beam divergence of $8^{\circ}$ (FWHM) for the VCSEL, the spot separation and the spot width becomes $0.16 \mathrm{~mm}$ and $0.06 \mathrm{~mm}$ at $L$ $=3 \mathrm{~mm}$, respectively. Therefore, we expect the relative drop in the signal obtained by the sensor using a transmitter DOE is caused by near field effects as the beams could overlap somewhat in the range from $L=1.5 \mathrm{~mm}$ to $3 \mathrm{~mm}$.

The applications for this technique are single-point measurements of in-plane translation, vibration and out-of-plane rotation of rigid bodies (e.g. in the backscattering configuration) or inplane fluid flow velocity (e.g. in the forward-scattering configuration). Compared with laser Doppler velocimetry and vibrometry methods, this technique offers a high degree of robustness, and compactness, while compared with traditional speckle translation methods it offers fast data processing. The robustness relies on the spatial filter, taking out the optical wavelength dependency of the power spectrum as $k \theta \rightarrow 2 \pi / \Lambda$ in (6) (when e.g. $\Lambda=\Lambda_{1}=\Lambda_{1}^{\prime}{ }_{1}$ ). With no additional optics in front of the spatial filter, the optics can be compressed into a cover plate [13] for an application-specificintegrated circuit (ASIC), which holds and drives the VCSEL, contains the photodetectors, the analogue electronics and the full digital signal processing, including peripheral communication, such as e.g. an universal serial bus device (USB). Therefore, one chip will contain the entire system for such an optical sensor.

The velocity (non relativistic) range of this technique is limited by the effective bandwidth of the electronic (analogue and digital) signal processing entirely. For example, in case of the spatial filter presented above a target moving with a velocity of $1 \mathrm{~km} / \mathrm{s}$ will require a bandwidth for the analogue electronics of at least $133 \mathrm{MHz}$, and a digital sampling rate running at least an order of magnitude faster $(1.5 \mathrm{~Gb} / \mathrm{s})$. Alternatively, the pitch of the lenticular array could be increased in order to reduce such speed requirements of the electronics.

\section{Conclusion}

This paper describes both theoretically and experimentally the advantage and limitations of seeding the speckle spectra with an adequate fine structure to match the spatial centre frequency of a spatial filtering velocimetry sensor. An in-line demonstrator illustrates the importance of matching the seeding frequency to the frequency of the spatial filter of the receiver. This setup illustrates the enhancement of the signal power by a factor of about 3-8, when replacing a single-beam illumination with three-beam illumination. With a similar backscatter system we have shown how to enhance the signal quality in terms of reducing an unwanted speckle signal, and the second harmonic of the fine structure.

The focus of this paper is the temporal power spectrum presented in (6), which has been compared favourable to experimental results for the photocurrent statistics, when combining speckle translation with an optical spatial filter.

\section{Acknowlegdement}

We acknowledge financial support from OPDI Technologies A/S, Denmark, and The Danish Foundation for Advanced Technology.

\section{References}

[1] T. Asakura, and N. Takai 1981, Dynamics laser speckles and their application to velocity measurements of the diffuse object", Appl. Phys., 25, 179-194.

[2] Aizu Y and Asakura T 1987, Principle and development of spatial filter velocimetry, Appl. Phys. B 43, 209-224.

[3] Tiziani H J 1972, A study of the use of laser speckle to measure small tilts of optically rough surfaces accurately, Opt. Commun. 5, 271-276. 
[4] Ator J T 1966, Image Velocity Sensing by Optical Correlation, Appl. Opt. 5, 1325-1331.

[5] Aizu Y and Asakura T 2006, Spatial Filtering Velocimetry, Fundamentals and Applications, Springer Series in Optical Science, Vol. 116, ISBN 3-540-28186-X.

[6] Ushizaka T and Asakura T 1983, Measurements of flow velocity in a microscopic region using a transmission grating, Appl. Opt. 22, 1870-1874.

[7] Aizu Y, Ushizaka T and Asakura T 1985, Measurements of the flow velocity in a microscopic region using a transmission grating: elimination of directional ambiguity, Appl. Opt. 24, 636640.

[8] Ushizaka T, Aizu Y and Asakura T 1986, Measurements of Velocity Using a Lenticular Grating, Appl. Phys. B 39, 97-106.

[9] Schnell U, Piot J and Dändliker R 1998, Detection of movement with laser speckle patterns: statistical properties, J. Opt. Soc. Am. A 15, 207-216.

[10] Yura H T, Hanson S G and Jakobsen M L 2008, Speckle dynamics resulting from multiple interfering beams, J. Opt. Soc. Am. A 25, 318-326.

[11] Jakobsen M L and Hanson S G 2008, Speckle dynamics for intensity-modulated illumination, Appl. Opt. 47, 3674-3680.

[12] Jakobsen M L and Hanson S G 2004, Lenticular array for spatial filtering velocimetry of laser speckles from solid surfaces, Appl. Opt. 43, 4643-4651.

[13] Hanson, S.G.; Jakobsen, M.L.; Petersen, H.C.; Dam-Hansen, C.; Stubager, J., Miniaturized optical speckle-based sensor for cursor control. In: Speckles, from grains to flowers. Proceedings. Speckle 06, Nîmes (FR), 13-15 Sep 2006. (International Society for Optical Engineering, Bellingham, 2006); SPIE Proceedings Series, 6341, 6341 1U) 